\title{
LES modelling of a flow within an infinite array of randomly placed cylinders
}

\section{Anisotropy characterization}

\author{
Ana M. Ricardo ${ }^{1, *}$, Dimokratis G.E. Grigoriadis ${ }^{2}$, and Rui M.L. Ferreira ${ }^{1}$ \\ ${ }^{1}$ CERIS, Instituto Superior Técnico, Universidade de Lisboa, Portugal \\ ${ }^{2}$ UCY-CompSci, Department of Mechanical and Manufacturing Engineering, University of Cyprus
}

\begin{abstract}
The LES approach is employed to model flows within random arrays of emergent cylinders. The model is validated against laboratory data acquired with a 2D-2C Particle Image Velocimetry system. The main goals are: i) discussion of the effect of the numerical domain size and the grid resolution on the predicted flow variables; and ii) spatial characterization of the flow anisotropy. Three domains of different sizes (16 to 36 cylinders) and four grid resolutions were independently tested. A 2D methodology was proposed to characterize the flow anisotropy on the horizontal plane. The results show that the first and second order moments were not significantly affected by the size of the tested numerical domains or by the grid resolution. The comparison with laboratory data showed a fair agreement confirming that the numerical model was able to adequately reproduce all the components of the Reynolds stress tensor. The results show that turbulence is of axisymmetric expansion nature in this type of flow. Relatively to the degree of anisotropy, the highest values were found close to the cylinder, decreasing gradually downstream towards the isotropy state. However, a truly isotropic turbulence state is not reached.
\end{abstract}

\section{Introduction}

Turbulent flows within random arrays of circular cylinders are relevant for several industrial and environmental applications [1-3]. In particular, the Reynolds stress anisotropy plays a fundamental role on the understanding of anisotropic turbulent kinetic energy production, the pressure-strain tensor relevance and the return to isotropy processes [4-6]. The quantification of all the Reynolds stress anisotropy tensor terms requires time series of the three velocity components. In complex geometries, as arrays of randomly placed cylinders, 3D laboratory or field measurements are extremely difficult of obtaining. But a complete three dimensional picture of the flow can be obtained from computational fluid dynamics models. The large eddy simulation (LES) approach is able of predicting an almost complete description of the instantaneous 3D turbulent flow field, resolving large-scale unsteadiness and asymmetries resulting from flow instabilities. Thus, LES has been very often employed to simulate flows within solid obstacles [2, 7-9].

In the present work the LES model developed by Grigoriadis et al. [7, 10] is employed to model flows within arrays of randomly placed emergent cylinders, as a proxy to large spatial

\footnotetext{
*e-mail: ana.ricardo@tecnico.ulisboa.pt
} 
domains of rigid vegetation. The model is validated against laboratory data acquired with a 2D-2C Particle Image Velocimetry system (PIV). The work aims at: i) discussing the effect of the numerical domain size and the grid resolution on the first and second order moments; and ii) characterizing the spatial distribution of flow anisotropy. Different domain sizes and grid resolutions were independently tested. This work mainly focuses on the drag-wake controlled layer, where the flow is controlled by the cylinders and the flow properties are approximately constant in the vertical direction [11].

Throughout this work, a Cartesian reference frame is considered, where $x, y$, and $z$ correspond to the streamwise, spanwise, and vertical directions, $u, v$, and $w$ are the corresponding velocity components respectively. Overbars $\left(\bar{u}_{i}\right)$ represent the time-average operator and primes $\left(u_{i}^{\prime}\right)$ the fluctuation relatively to the time-averaged variable. The flow variables are presented dimensionless, turbulence intensities are defined by the root-mean-square of the velocity fluctuation and shear stresses are considered as stresses per unit mass.

\section{Laboratory test}

The experimental work was carried out in a $12.5 \mathrm{~m}$ long and $41 \mathrm{~cm}$ wide recirculating tilting flume with glass side walls. The flume bottom was horizontal and covered with an horizontal layer of gravel $\left(D_{50}=7.5 \mathrm{~mm}\right)$ and sand $\left(D_{50}=0.8 \mathrm{~mm}\right)$. Rigid, slightly rough, vertical and circular cylinders were placed along of a $3.5 \mathrm{~m}$ long reach with cylinder areal numberdensity of 980 cylinders $/ \mathrm{m}^{2}$. The location of the cylinders was defined by a random function obeying to spatial uniformity. A measuring gap was enforced, whose width is equal to the mean inter-cylinder distance (Fig. 1). The flow was steady and gradually accelerated with a depth gradient of $\mathrm{d} h / \mathrm{d} x=-0.014$, flow depth $h=5.1 \mathrm{~cm}$ and mean longitudinal velocity $U_{0}=0.099 \mathrm{~ms}^{-1}$ in the measuring gap. The Reynolds number, defined as $R e_{d}=U_{0} d / v(d$ is the cylinder diameter and $v$ is the water kinematic viscosity) was equal to 1064 .

Measurements consisted in acquisition of 2D instantaneous velocity maps with a PIV system composed by an 8 -bit $1600 \times 1200 \mathrm{px}^{2} \mathrm{CCD}$ camera and a double-cavity Nd-YAG laser with pulse energy of $30 \mathrm{~mJ}$ at wavelength of $532 \mathrm{~nm}$. PIV image pairs were acquired at a frequency of $15 \mathrm{~Hz}$ with a time delay of $1500 \mu$ s between frames. Polyurethane particles with a mean diameter of $60 \mu \mathrm{m}$ and a density of $1.31 \mathrm{~g} / \mathrm{cm}^{3}$ were employed as seeding material. For details on the PIV system and seeding particles employed see [12]. The instantaneous velocity maps were acquired at eight vertical planes (Fig. 1) and one horizontal $(z / d=$ 3.8). Each acquisition records consecutively 5000 image pairs and the field of view was $\approx 12 \times 10 \mathrm{~cm}^{2}$, corresponding to interrogation areas of $\approx 1.2 \times 1.2 \mathrm{~mm}^{2}$.

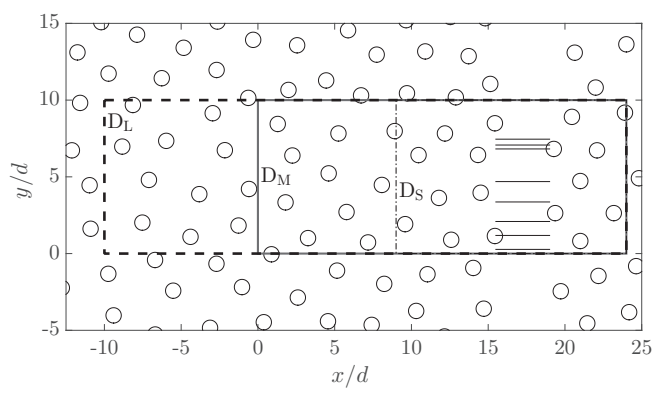

Figure 1. Part of the cylinder distribution. The solid lines aligned with flow direction (left to right) identify the location of the acquired vertical planes. The rectangles represent the numerical domains. 


\section{Numerical model}

The model employed $[7,8,10]$ was designed for efficient, parallel, three-dimensional computations of the Navier-Stokes equations in complex geometrical configurations. It is based on a fully explicit, fractional step approach and finite differences on staggered, Cartesian grids. Time advancement is performed using a fully explicit second order Adams-Bashforth scheme, and the momentum equation is discretized with a central, finite difference scheme.

The cylinder boundaries are described by the immersed boundary method. At this part of the computational domain, the solution is locally reconstructed in the vicinity of the boundaries, since the cylinders are not aligned with the grid lines [7, 8].

Three numerical setups were tested, changing the streamwise extend of the domain $\left(L_{x}\right)$, as shown in Table 2. The vertical size of the domain, $\left(L_{z}\right)$ and the width $\left(L_{y}\right)$ were kept constant. The relative location of the cylinders, in all numerical simulations, corresponds exactly to the location in the laboratory setup (Fig. 1). The numerical domain boundaries were set so that the application of periodic boundary condition reproduces as much as possible the laboratory conditions.

The bottom is defined by an horizontal smooth wall and at the top a rigid lid was employed to fix the flow depth. A non-slip condition was applied along the bed and periodic boundary conditions are used along $x-y$ directions. Along the free-surface a non-slip boundary condition was employed with $\mathrm{d} U / \mathrm{d} z=0, \mathrm{~d} V / \mathrm{d} z=0$ and $W=0$. The simulations were run in non-dimensional conditions where distances are scaled with the cylinder diameter $d=11 \mathrm{~mm}$, velocities with $U_{0}=0.099 \mathrm{~ms}^{-1}$, leading to a characteristic time scale of $t_{0}=d / U_{0}=0.111 \mathrm{~s}$. When the solution reaches a statistically steady state, statistics were collected over a time interval of approximately $500 \mathrm{~d} / U_{0}$.

\section{Effect of grid resolution}

To assess the effect of the grid size on the predicted flow fields, four different grids were employed to model the medium size domain $\mathrm{D}_{\mathrm{M}}$ (Table 2), which corresponds to a box with dimensions $24 d \times 10 d \times 4.64 d$ (Fig. 1). The parameters of the tested grids are summarize in Table 1. Figure 2 compares horizontal and vertical profiles of time-averaged lateral velocity and longitudinal and vertical turbulence intensity computed with different grid sizes. Globally, the comparison of flow variables computed with the different meshes reveals that the effect of the grid resolution on the magnitude of the simulated variables is relatively small for the meshes tested, and the impact on the turbulence intensities being larger than on the velocity field. Quantitatively, the square differences for any combination of meshes showed bounded values within order of magnitude $10^{-3}$ or smaller. Moreover, the results show that the numerically computed flow variables are fairly close to the laboratory variables. Despite the results for all the tested meshes are similar, grid G1 presents the largest differences relatively to the other meshes and to the laboratory data. Although the results obtained by the grids G3 and G4 do not overlap completely, they are closer to the laboratory data than the coarsest grids G1 and G2.

Table 1. Parameters of the tested grids.

\begin{tabular}{ccccc}
\hline Grid & Resolution & Min cell from bed $(\mathrm{mm})$ & $d \times d$ (cells) & Time step $(\mathrm{ms})$ \\
\hline G1 & $512 \times 256 \times 65$ & 0.418 & $22 \times 25$ & 2.3 \\
G2 & $768 \times 384 \times 97$ & 0.220 & $32 \times 38$ & 1.7 \\
G3 & $1024 \times 512 \times 129$ & 0.110 & $43 \times 51$ & 1.2 \\
G4 & $1536 \times 768 \times 192$ & 0.077 & $64 \times 76$ & 0.8 \\
\hline
\end{tabular}




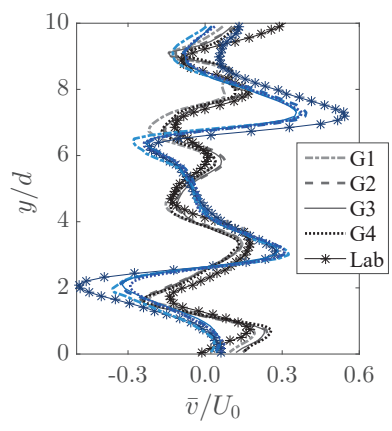

(a)

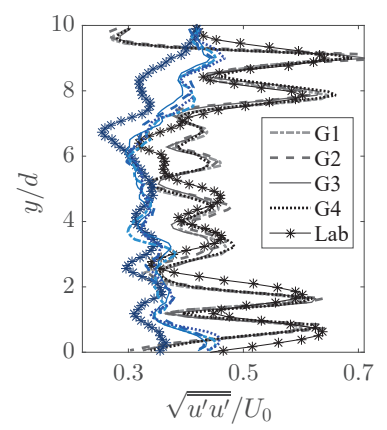

(b)

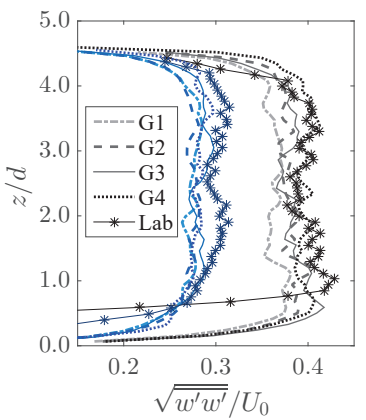

(c)

Figure 2. Time-averaged profiles: a) lateral velocity (gray: $x / d=16.5, z / d=3.8$; blue: $x / d=18.5$, $z / d=3.8$ ); b) longitudinal turbulence intensity (gray: $x / d=16.5, z / d=3.8$; blue: $x / d=18.5, z / d=3.8$ ); c) vertical turbulence intensity (gray: $x / d=16.5, y / d=1.4$; blue: $x / d=18.5, y / d=2.1$ ).

\section{Effect of domain size}

To discuss the impact of the number of cylinders modelled on the flow variables, three different domains were simulated, $D_{S}, D_{M}$, and $D_{L}$, as shown in Figure 1. Table 2 summarises the characteristics of the tested setups. The intermediate grid resolution G2 was employed.

Figure 3 displays time-averaged lateral profiles of first and second order moments for each tested domain, sampled at two longitudinal positions at $z / d=3.8$. Comparing the profiles,

Table 2. Features of the computational domains and the time to compute one second of the flow $\left(T_{s}\right)$.

\begin{tabular}{cccccc}
\hline & Resolution & Size & Nr. Cylind. & Time step $(\mathrm{ms})$ & $T_{S}(\mathrm{~min})$ \\
\hline $\mathrm{D}_{\mathrm{S}}$ & $480 \times 384 \times 97$ & $15 \times 10 \times 4.636$ & 16 & 0.31 & 30 \\
$\mathrm{D}_{\mathrm{M}}$ & $768 \times 384 \times 97$ & $24 \times 10 \times 4.636$ & 26 & 0.21 & 50 \\
$\mathrm{D}_{\mathrm{L}}$ & $1080 \times 384 \times 97$ & $33.75 \times 10 \times 4.636$ & 36 & 0.15 & 80 \\
\hline
\end{tabular}

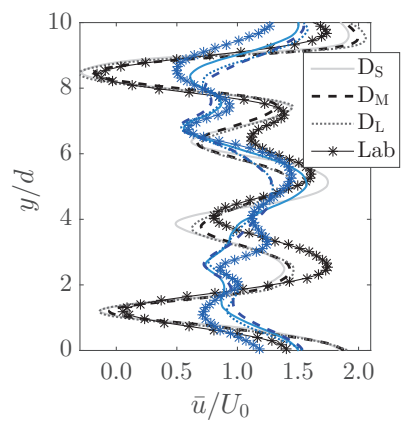

(a)

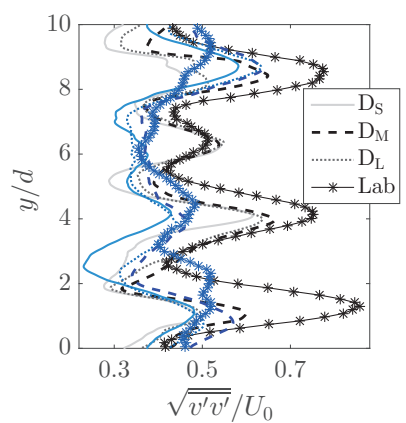

(b)

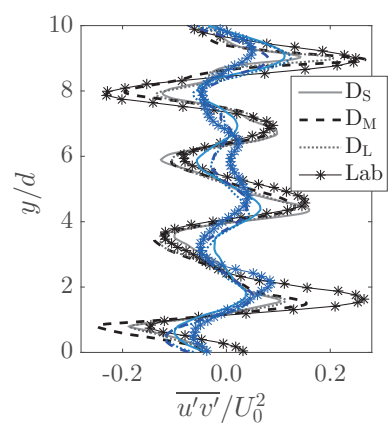

(c)

Figure 3. Time-averaged lateral profiles of: a) longitudinal velocity; b) longitudinal turbulence intensity and c) longitudinal-lateral shear stress, sampled at $z / d=3.8$ and $x / d=16.5$ (gray) and $x / d=18.5$ (blue). 
one observes that $\mathrm{D}_{M}$ and $\mathrm{D}_{\mathrm{L}}$ profiles are closer to each other and exhibit a relatively larger distance to $\mathrm{D}_{\mathrm{S}}$ profiles. Although the differences are small, laboratory data profiles are closer to the profiles of $D_{M}$ and $D_{L}$ than of $D_{S}$.

Globally, the comparison of flow variables modelled by the setups of different size reveals that the flow variables are not significantly affected by the domain size, as relevant differences were not detected. Furthermore, the numerically modelled flow variables are fairly close to those computed from the laboratory database. The present work brings evidence that it is possible to accurately forecast the first order moments setting the domain to the largest size ensuring a reasonable simulation time. Fair estimates of the second order moments are also possible, however losses in quality are expected on the background turbulence levels.

\section{Spatial characterization of Reynold stresses}

In this section the numerical database is employed as an extension of the laboratory database to characterize spatially the Reynold stress tensor components, in the drag-wake controlled layer. The comparison of numerical and laboratory data will also be further analysed. The numerical data employed herein was obtained with the domain $\mathrm{D}_{\mathrm{M}}$ and the grid $\mathrm{G} 4$.

Figure 4 presents spatial distributions of longitudinal and lateral turbulence intensities as well as the longitudinal-lateral shear stress, from numerical and laboratory databases. The pattern of the distribution and the magnitude of the variables are fairly well predicted by the

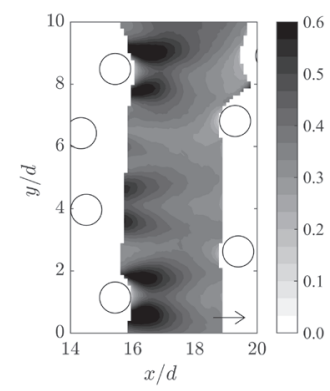

(a) $\operatorname{Exp} \sqrt{\overline{u^{\prime} u^{\prime}}} / U_{0}$

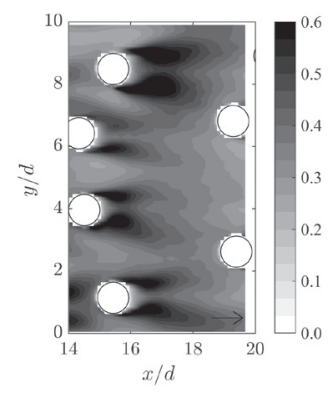

(d) Num $\sqrt{\overline{u^{\prime} u^{\prime}}} / U_{0}$

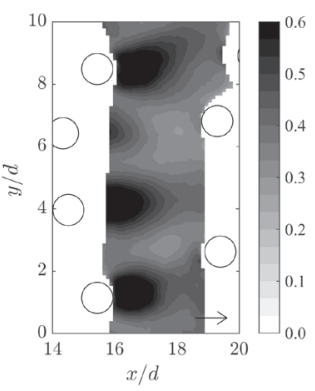

(b) $\operatorname{Exp} \sqrt{\overline{v^{\prime} v^{\prime}}} / U_{0}$

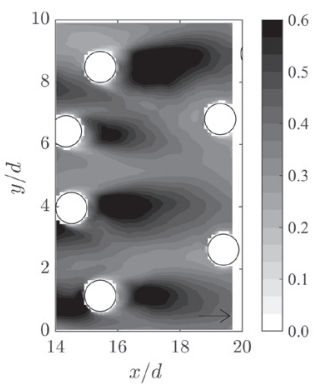

(e) Num $\sqrt{\overline{{v^{\prime} v^{\prime}}^{\prime}}} / U_{0}$

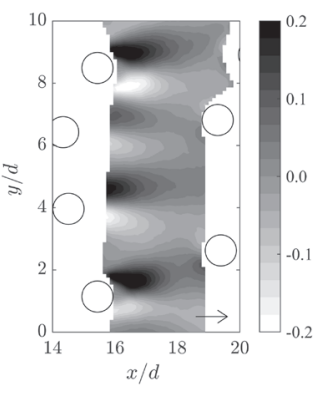

(c) $\operatorname{Exp} \overline{u^{\prime} v^{\prime}} / U_{0}^{2}$

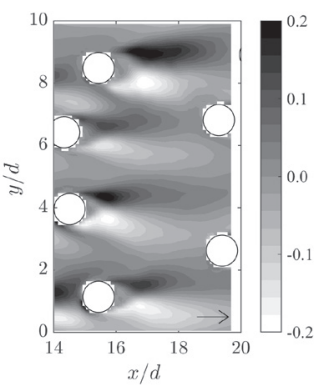

(f) $\operatorname{Num} \overline{u^{\prime} v^{\prime}} / U_{0}^{2}$

Figure 4. Two-dimensional maps of laboratory (top: a-c) and numerical (bottom: d-f) variables at $z / d=3.8$ : a) and d) longitudinal turbulence intensity; b) and e) lateral turbulence intensity; c) and f) longitudinal-lateral turbulent shear stress. The arrow indicates the flow direction. 


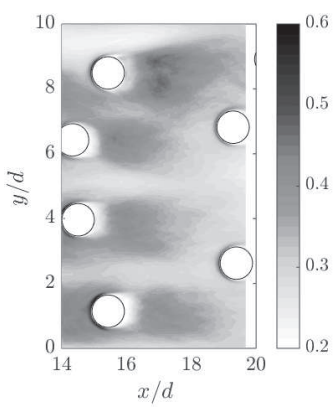

(a) $\sqrt{\overline{\overline{w^{\prime} w^{\prime}}}} / U_{0}$

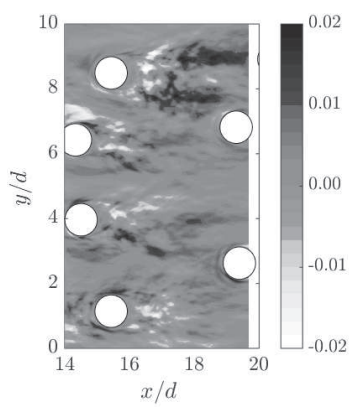

(b) $\overline{u^{\prime} w^{\prime}} / U_{0}^{2}$

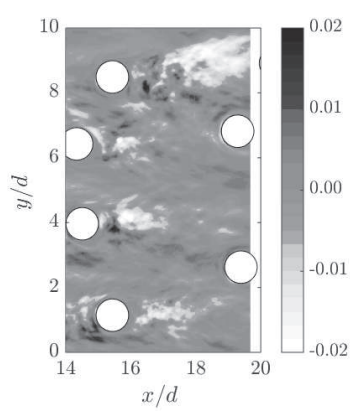

(c) $\overline{v^{\prime} w^{\prime}} / U_{0}^{2}$

Figure 5. Two-dimensional maps at $z / d=3.8$ : a) vertical turbulence intensity; b) longitudinal-vertical turbulent shear stress; and c)lateral-vertical turbulent shear stress.

model. The turbulence intensities out of the near wake of the cylinders are slightly smaller in the numerical tests indicating that background turbulence is weaker. Another difference is the location of the variables maximum magnitude relatively to each cylinder: in the laboratory database the highest values are found closer to the cylinder and they decay faster than in the simulations. Nevertheless, the lateral variation is accurately predicted, i.e., the peaks and troughs are found at the same $y$ - location both in the laboratory and numerical domains.

Figure 5 shows the spatial distribution of $\sqrt{\overline{w^{\prime} w^{\prime}}}, \overline{u^{\prime} w^{\prime}}$ and $\overline{v^{\prime} w^{\prime}}$ numerically computed.

The spatial distribution of the longitudinal turbulence intensity, presented in Figure 4 (a and d), shows the largest magnitudes on the vortex street downstream of each cylinder and lower values in the corridors between cylinders. While the lateral (Fig. $4 \mathrm{~b}$ and e) and vertical (Fig. 5a) turbulence intensities are stronger on the wake of each cylinder. These patterns are similar to the symmetric distribution of turbulence intensities of a flow around a single cylinder. Within the array the symmetry is disrupted by the presence of the neighbouring cylinders. Regions with almost zero turbulence intensities, observed in the regular distributions of cylinders [2], practically do not exist in random distributions. Figures 4(a-d) and 5(a), corroborating with Ricardo et al [3], indicate that the randomness of array promotes an efficient generation of background turbulence, relatively to the case of regular distributions.

Figures 4(c) and (f) present the non-dimensional longitudinal-lateral component of the Reynolds shear stresses, $\overline{u^{\prime} v^{\prime}} / U_{0}^{2}$, showing an almost anti-symmetric pattern relatively to the axis of each cylinder of high positive and negative values on the wake of each cylinder, and almost zero values between cylinders. The longitudinal-vertical and lateral-vertical components of the turbulent shear stress, Figures 5(b) and (c), are much smaller than $\overline{u^{\prime} v^{\prime}} / U_{0}^{2}$, exhibiting the largest magnitudes within the cylinders wake.

\section{Anisotropy}

The study of anisotropic turbulence is of great interest on the understanding of the turbulent kinetic energy production, the pressure-strain tensor relevance, the return to isotropy processes and also on the development of turbulent small scale models [5, 6]. The most common methodology on the study of the Reynolds stress anisotropy is the Lumley triangle [13] (or anisotropic invariant map). It consists of a partially curvilinear triangle, in a coordinate system defined by the second and the third invariants of the anisotropy tensor, which encloses all feasible flow realisations and determines the anisotropy state of the flow. To improve the 


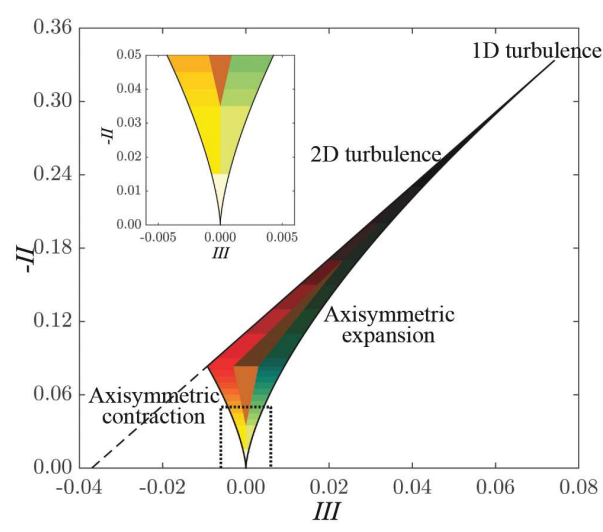

(a) Anisotropy color mapping

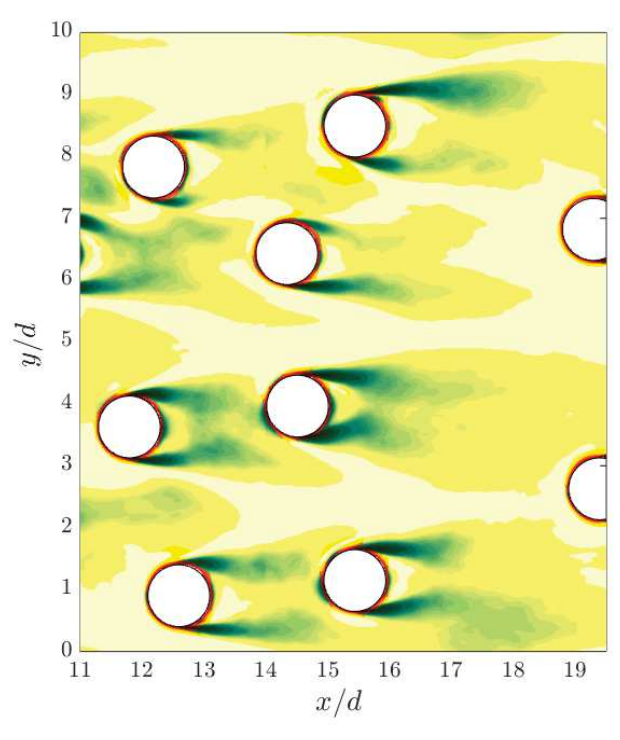

(b) $2 \mathrm{D}$ anisotropy map

Figure 6. $2 \mathrm{D}$ distribution of the Reynolds stress tensor anisotropy at $z / d=3.8$.

spatial characterization the Reynolds stress tensor anisotropy, a new method based on the traditional Lumley plot is explored herein. This consists in representing the Lumley triangle as a color pattern and employing such distribution as color mapping for a $2 \mathrm{D}$ representation of the anisotropy states, as illustrated in Figure 6(a). In that way the invariant pair $(I I I,-I I)$ of each point of the 2D space is associated to a color identifying its location within the Lumley triangle. Representing the anisotropy invariants in this fashion allows for a simultaneous spatial characterization of the nature and the degree of the flow anisotropy.

Figure 6(b) shows that the flow within an array of cylinders is characterized by cigarshaped turbulence (axisymmetric expansion turbulence). Figure 6(b) suggests that the flow is less anisotropic in regions where the turbulent kinetic energy and its production are reduced [3]. The anisotropy reduces with the downstream distance to the cylinders and the most isotropic regions are found along corridors relatively distant from the cylinders. Very close to the surface of the cylinders, a 2D turbulence state can be identified. Although the longitudinal normal Reynolds stress $\left(\overline{u^{\prime} u^{\prime}}\right)$ is expected to vanish on the cylinder boundary, the distribution of anisotropy on the close vicinity of the cylinders in Figure 6(b) might be a consequence of the velocity filed reconstruction required by the immersed boundary method employed in the simulations. The anisotropy distribution suggests that the symmetry pattern in the wake of a single cylinder is impacted by local distribution of the neighbouring cylinders.

The highest degree of anisotropy is found on the side of the cylinders where the vortex streets begin. In these regions $\overline{u^{\prime} u^{\prime}}$ (Fig. $4 \mathrm{a}$ and d) is larger than $\overline{v^{\prime} v^{\prime}}$ and $\overline{w^{\prime} w^{\prime}}$ (Fig. $4 \mathrm{~b}$ and e and 5a). Further downstream the magnitude of $\overline{u^{\prime} u^{\prime}}$ decreases to values close to $\overline{w^{\prime} w^{\prime}}$, while $\overline{v^{\prime} v^{\prime}}$ increases and becomes the highest normal component of the Reynolds stress tensor. The turbulence keeps its axisymmetric expansion shape but the degree of anisotropy decreases gradually downstream. On the wake centreline, the anisotropy degree is relatively small on the very near wake, where all the normal components of the turbulent stress are close to zero. 
Towards downstream there is an increase of the anisotropy degree before a gradual decrease that is also observed on the magnitude of TKE [3].

Overall, the anisotropy analysis shows a tendency toward isotropy, however a truly isotropic turbulence state seems not achievable for random arrays of cylinders with the same or larger number of cylinders per unit area of the presented test. Such a finding suggests that employing return-to-isotropy approaches to model the evolution of the Reynolds stress tensor of flows within arrays of bluff bodies requires special attention.

\section{Conclusion}

Numerical simulations were performed and compared against a laboratory test of a fully developed flow around randomly placed emergent circular cylinders. Four different grid resolutions were tested revealing similar results, however the coarsest grid presented consistently the largest differences. The discussion of the impact of the number of cylinders modelled reveal no significant effect of domain size on the the time-averaged velocity field and the Reynolds stress tensor. This indicates that the first and second order moments of flows in infinite domains of randomly placed cylinders can be accurately predicted by LES modelling with a relatively small domain and periodic boundary conditions.

The numerical model proved to be able to reproduce the flow in the drag-wake controlled layer; the numerical flow variables were found in a fair agreement with the laboratory data.

A 2D methodology was proposed to characterize the flow anisotropy on the horizontal plane. The results showed that the drag-wake controlled layer is characterized by cigar-shaped turbulence (axisymmetric expansion turbulence). Relatively to the degree of anisotropy, the highest values were found on the side of the cylinders where the vortex streets begin, decreasing gradually with the downstream distance to the cylinder towards the isotropy turbulence state. However, a truly isotropic turbulence state was not reached.

\section{Acknowledgements}

This work was partially funded] by FEDER, program COMPETE, and by national funds through Portuguese Foundation for Science and Technology (FCT) project PTDC/ECMHID/6387/2014 and by the grant SFRH/BPD/93903/2013.

\section{References}

[1] D. Sumner, J.Fluid. Struct. 26, 849 (2010)

[2] T. Stoesser, S. Kim, P. Diplas, J. Hydraul. Eng. 136, 1003 (2010)

[3] A.M. Ricardo, K. Koll, M.J. Franca, A.J. Schleiss, R.M. Ferreira, Water Resour. Res. 50, 4131 (2014)

[4] R. Antonia, P.Å. Krogstad, Fluid Dyn. Res. 28, 139 (2001)

[5] R. Smalley, S. Leonardi, R. Antonia, L. Djenidi, P. Orlandi, Exp. Fluids 33, 31 (2002)

[6] A. Ashrafian, H.I. Andersson, Int. J. Heat Fluid Fl. 27, 65 (2006)

[7] D.G.E. Grigoriadis, J.G. Bartzis, A. Goulas, Int. J. Numer. Meth. Fl. 41, 615 (2003)

[8] D.G.E. Grigoriadis, E. Balaras, A.A. Dimas, Flow Turbul. Combust. 91, 565 (2013)

[9] W. Rodi, J. Hydraul. Eng. 143, 03117001 (2017)

[10] D. Grigoriadis, J. Bartzis, A. Goulas, Comput. Fluids 33, 201 (2004)

[11] A.M. Ricardo, M.J. Franca, R.M. Ferreira, J. Hydraul. Eng. 142, 04016022 (2016)

[12] A.M. Ricardo, Ph.D. thesis, Instituto Superior Técnico \& EPFLausanne (2014)

[13] J.L. Lumley, G.R. Newman, J. Fluid Mech. 82, 161 (1977) 